\title{
Adaptation to visual numerosity changes neural numerosity selectivity
}

\author{
Andromachi Tsouli ${ }^{\mathrm{a}, *}$, Yuxuan Cai ${ }^{\mathrm{b}, \mathrm{c}}$, Martijn van Ackooij ${ }^{\mathrm{a}}$, Shir Hofstetter ${ }^{\mathrm{b}}$, Ben M. Harvey ${ }^{\mathrm{a}}$, \\ Susan F. te Pas ${ }^{\mathrm{a}}$, Maarten J. van der Smagt ${ }^{\mathrm{a}}$, Serge O. Dumoulin ${ }^{\mathrm{a}, \mathrm{b}, \mathrm{c}}$ \\ ${ }^{a}$ Department of Experimental Psychology, Helmholtz Institute, Utrecht University, Heidelberglaan 1, Utrecht, CS 3584, Netherlands \\ ${ }^{\mathrm{b}}$ The Spinoza Centre for Neuroimaging, Amsterdam, Netherlands \\ ${ }^{\mathrm{c}}$ Department of Experimental and Applied Psychology, VU University, Amsterdam, Netherlands
}

\section{A R T I C L E I N F O}

\section{Keywords:}

Numerosity

Adaptation

Topographic maps

High-field 7T fMRI

\begin{abstract}
A B S T R A C T
Perceiving numerosity, i.e. the set size of a group of items, is an evolutionarily preserved ability found in humans and animals. A useful method to infer the neural underpinnings of a given perceptual property is sensory adaptation. Like other primary perceptual attributes, numerosity is susceptible to adaptation. Recently, we have shown numerosity-selective neural populations with a topographic organization in the human brain. Here, we investigated whether numerosity adaptation can affect the numerosity selectivity of these populations using ultra-high field (7 Tesla) functional magnetic resonance imaging (fMRI). Participants viewed stimuli of changing numerosity (1 to 7 dots), which allowed the mapping of numerosity selectivity. We interleaved a low or high numerosity adapter stimulus with these mapping stimuli, repeatedly presenting 1 or 20 dots respectively to adapt the numerosity-selective neural populations. We analyzed the responses using custom-build population receptive field neural models of numerosity encoding and compared estimated numerosity preferences between adaptation conditions. We replicated our previous studies where we found several topographic maps of numerosity-selective responses. We found that overall, numerosity adaptation altered the preferred numerosities within the numerosity maps, resulting in predominantly attractive biases towards the numerosity of the adapter. The differential biases could be explained by the difference between the unadapted preferred numerosity and the numerosity of the adapter, with attractive biases being observed with higher difference. The results could link perceptual numerosity adaptation effects to changes in neural numerosity selectivity.
\end{abstract}

\section{Introduction}

The ability to process numerosity, i.e. the set size of a group of items, is an essential neurobiological feature found in humans from the moment of birth (Izard et al., 2009), and other species such as nonhuman primates (Nieder et al., 2002), birds (Scarf et al., 2011), amphibians (Krusche et al., 2010), fish (Miletto Petrazzini et al., 2016), and insects (Howard et al., 2018). Converging evidence from electrophysiology, psychophysics, and functional neuroimaging supports the existence of neural populations responding to specific numerosity ranges. More specifically, single-cell activity recordings in humans (Kutter et al., 2018), macaques (Nieder and Miller, 2003, 2004; Viswanathan and Nieder, 2013), and crows (Wagener et al., 2018) have revealed neurons which respond selectively to low numerosities. The response amplitude of these neurons peaks when a specific numerosity is presented, i.e. the preferred numerosity, and decreases with the difference between the logarithm of the preferred numerosity and the presented numerosity.

Neural numerosity selectivity has also been examined using psychophysics and adaptation paradigms. Perceptual adaptation paradigms repeatedly present a particular stimulus (the adapter), which makes subsequently presented (reference) stimuli appear more different from the adapter than they are. Numerosity, similarly to other visual properties such as color or contrast, is susceptible to adaptation, yielding 'repulsive' aftereffects (see review by Anobile et al., 2016). Specifically, adaptation to a low numerosity leads to an overestimation of the numerosity subsequently presented in the adapted location, whereas adaption to a high numerosity leads to an underestimation (Burr et al., 2017; Burr and Ross, 2008). This perceptual repulsion from the adapter is often taken to demonstrate tuned neural responses to the adapted stimulus property because it is hypothesized to reduce the response amplitude of part of the neural tuning function, pushing the preferred stimulus state away from the adapter. Very low numerosities (as low as 3) can also be affected by adaptation, albeit with manipulation of attentional resources (Burr et al., 2011). Moreover, the aftereffects produced by adaptation to numerosity can occur even when using few trials and a brief presentation of the adapting numerosity stimulus (Aagten-Murphy and Burr, 2016; Tsouli et al., 2019a,b).

\footnotetext{
* Corresponding author.

E-mail address: a.tsouli@uu.nl (A. Tsouli).
} 
Functional magnetic resonance imaging (fMRI) adaptation paradigms have also been used to elucidate neural selectivity to numerosity (Cantlon et al., 2006; Demeyere et al., 2014; Roggeman et al., 2011; Shuman and Kanwisher, 2004). This method takes advantage of the decreased blood-oxygenation-level-dependent (BOLD) responses associated with the repeated presentation of a specific stimulus, with responses recovering when a different stimulus is subsequently presented (Grill-Spector et al., 2006; Krekelberg et al., 2006; Larsson et al., 2016). For numerosity, the extent of this response recovery follows the numerical difference between the adapter and reference stimuli, suggesting neural tuning for numerosity (He et al., 2015; Jacob and Nieder, 2009; Kersey and Cantlon, 2017; Piazza et al., 2004). Furthermore, a study examining the effect of numerosity adaptation on numerosity decoding using fMRI and multi-voxel pattern analysis (MVPA) found less accurate (indeed chance level) classification of numerosities after adaptation when classifiers were trained on pre-adaptation data (Castaldi et al., 2016).

We have previously shown topographically organized neural populations tuned to numerosity, primarily located in and around the post-central sulcus in the superior parietal lobe, using ultra-high field (7 Tesla) fMRI and population receptive field (pRF) modeling (Harvey et al., 2013; Harvey and Dumoulin, 2017a). We further showed that the numerosity selectivity of these neural populations is relatively independent from non-numerical visual features (Harvey et al., 2015; Harvey and Dumoulin, 2017a,b).

The goal of the present study was to examine whether the tuning of these numerosity selective neurons is systematically altered during perceptual adaptation following the repeated presentation of specific numerosities. Participants viewed stimuli of changing numerosity to map numerosity selectivity, as in our previous studies (control condition). In the experimental conditions, these numerosities were interleaved with a low numerosity (1 dot) or high numerosity (20 dots) adapter. We hypothesized that if neural populations' numerosity tuning is affected by adaptation, then their preferred numerosity would change depending on both the presence and the numerosity of the adapter.

\section{Methods}

\subsection{Participants}

We present data from eight human participants (five male, three female; age range 26-52 years). One was left-handed. All were well educated (postgraduate), with good mathematical abilities, and had normal or corrected-to-normal visual acuity. All gave written informed consent. All experimental procedures were approved by the ethics committee of University Medical Center Utrecht (protocol number 09/350).

\subsection{Numerosity stimuli}

The numerosity stimuli were presented on a $69.84 \times 39.29 \mathrm{~cm}$ LCD screen (Cambridge Research Systems) positioned behind the MRI bore. Participants were required to lie still and view the display through a mirror attached to the head coil. The total distance from the attached mirror to the display screen was $220 \mathrm{~cm}$ and the display resolution was $1920 \times 1080$ pixels.

The stimuli were generated and presented using MATLAB (MathWorks, Inc.) and the Psychophysics Toolbox (Brainard, 1997; Kleiner et al., 2007). Similarly to the methodology used in our previous studies (Harvey et al., 2013, 2015; Harvey and Dumoulin, 2017a,b), a large diagonal cross of thin red lines crossed the entire display, which facilitated accurate fixation at the cross intersection. The numerosity stimuli were groups of dots randomly positioned at each presentation so that all dots fell entirely within $0.75^{\circ}$ (radius) of fixation. The random position of the dots ensured that the contrast energy was distributed similarly across the stimulus area for all numerosities. Moreover, the individual dots were distributed roughly homogeneously across the stim- ulus area to avoid perceptual grouping. We kept the total surface area of all of the dots combined constant across numerosities which ensured equal luminance across numerosities.

In all conditions, the numerosities 1 through 7 and 20 were presented as black dots on a gray background in $90 \%$ of dot presentations (including the adapter), while in the remaining $10 \%$, the dots were shown in white (Fig. 1a). Each numerosity stimulus was presented briefly ( $300 \mathrm{~ms}$ ) to ensure participants did not have time to sequentially count the objects, at least in the case of higher numerosities (i.e. outside the subitizing range, $>4$ items). The numerosity stimulus was then followed by an interstimulus interval (ISI) of $400 \mathrm{~ms}$ showing a uniform gray background. In the control condition, and for numerosities 1 through 7 (the 'main' stimuli for quantifying numerosity tuning), this was repeated six times over $4200 \mathrm{~ms}$ (three fMRI volume acquisitions, TRs) to produce strong fMRI responses and facilitate the measurement of response tuning (Fig. 1b). Each of the main 1 through 7 numerosity stimuli was shown for a total of 48 times in each functional run. Each numerosity stimulus presentation contained a new random dot pattern, whether the numerosity changed or not.

In the low and high numerosity adaptation conditions, the main numerosity stimuli 1 through 7 were interleaved with a low $(1 \mathrm{dot})$ and high (20 dots) numerosity adapter respectively (Fig. 1a). More specifically, the main numerosity stimuli were first presented for $300 \mathrm{~ms}$, followed by a $400 \mathrm{~ms}$ ISI, followed by the numerosity adapter for $300 \mathrm{~ms}$, followed by another $400 \mathrm{~ms}$ ISI (Fig. 1b). This was repeated three times over $4200 \mathrm{~ms}$ (three TRs). Therefore, the adapter was presented before and after the main numerosity stimuli during the stimulus sequence. Each of the main 1 through 7 numerosity stimuli was shown for a total of 24 times and the numerosity adapter for a total of 24 times in each functional run. This design kept the timing of the main numerosity stimuli the same for all conditions. The control condition can be seen as a special case where the adapter followed the numerosity of the main stimulus, changing to minimize systematic adaptation effects: in all conditions the main numerosity stimulus is likely to cause some adaptation as it contains repeated presentations of the same numerosity.

In all conditions, the main numerosity stimuli were first presented in ascending order, followed by a longer period ( $16.8 \mathrm{~s}$ ) where the stimulus contained 20 dots, followed by the numerosities in descending order, followed by another long period of 20 dots. This sequence was repeated four times in each scanning run.

The long period of 20 dots served a relative "baseline" function, allowing us to distinguish neural populations with very small tuning widths which never responded to the main numerosities 1 through 7 , and populations with very large tuning widths which always responded to these numerosities (Harvey et al., 2013). Thus, during this period, relatively little neural response was expected from neurons with lower numerosity preferences, because a numerosity of 20 dots should be well outside of the range that elicits strong responses. This allows hemodynamic responses to return back to baseline between blocks of changing numerosity. In the low and high numerosity adaptation conditions, the long period of 20 dots was also interleaved with the low (1 dot) and high (20 dots) numerosity adapter respectively, to maintain adaptation.

Participants were instructed to press a button when the dots were shown in white instead of black in order to ensure that they were paying attention to the stimuli during fMRI acquisition. No numerosity judgments were required.

\subsection{Stimulus validation}

Given the fast, sequential stimulus presentation and the rapid alternation between the main numerosity stimuli and the adapters in our fMRI paradigm, we evaluated whether our stimulus sequence does indeed yield repulsive aftereffects behaviorally (Aagten-Murphy and Burr, 2016; Tsouli et al., 2019a,b). In the behavioral validation, we used the same numerosity adapters (1 and 20) and evaluated their effect on a reference stimulus of 10 dots, using a numerosity discrimination task 
a.

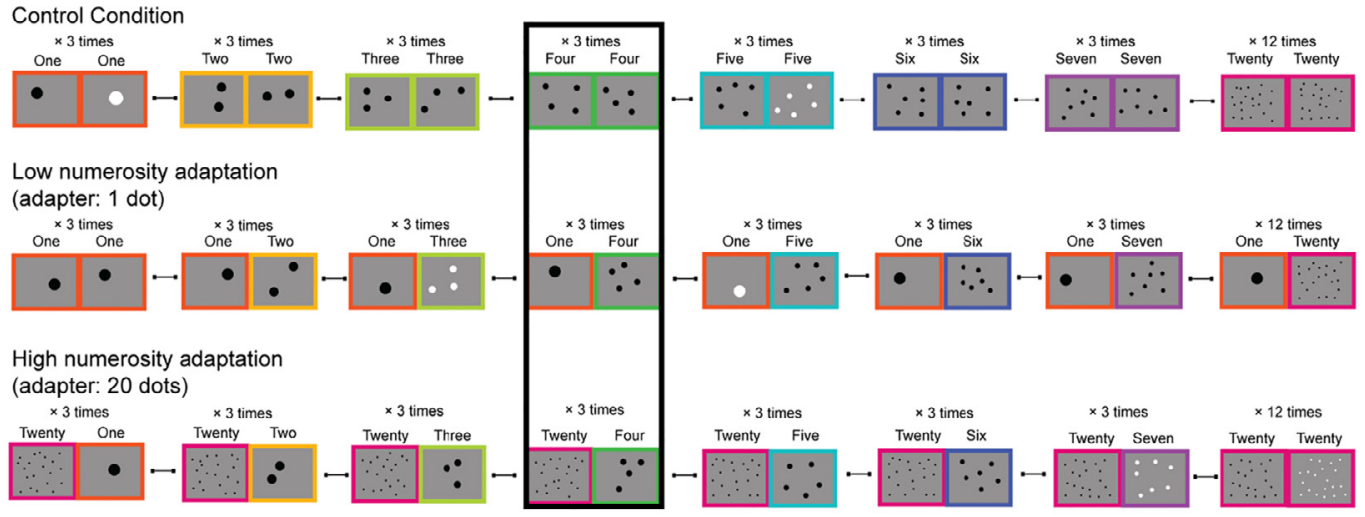

b. Control Condition
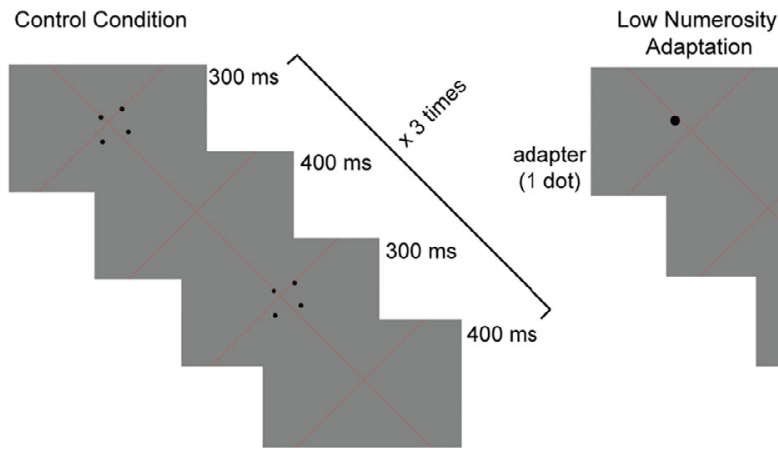
Adaptation
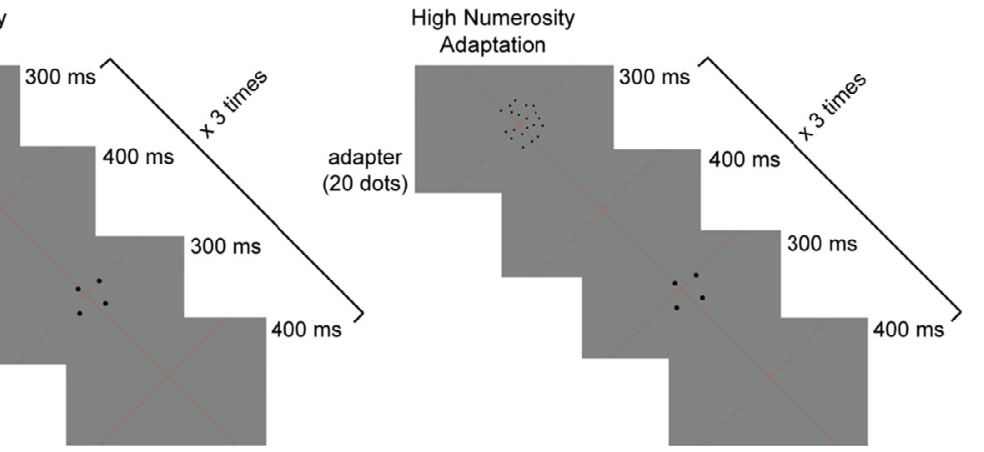

Fig. 1. Example of numerosity stimuli used (a) and schematic representation of stimuli presentation in each condition (b). (a) In the control condition, numerosities 1 through 7 were the main numerosity stimuli, followed by a baseline of 20 dots. In the adaptation conditions, the main numerosity stimuli and baseline were interleaved with a low (1 dot) or high (20 dots) numerosity adapter. Therefore, the adapter was presented before and after the main numerosity stimuli during the stimulus sequence. (b) In the control condition, each of the main numerosity stimuli was shown six times before the numerosity changed, to ensure strong fMRI responses. In the adaptation conditions, the main numerosity stimuli and the adapter were shown three times before the numerosity changed, to ensure that changes in the main stimulus had the same timing in the adaptation conditions and control condition.

(see Supplementary Materials for more details). We used a reference stimulus of 10 dots since the behavioral detection of numerosity adaptation effects on very low numerosities (within the subitizing range) appears to require the introduction of an attention-demanding secondary task (Burr et al., 2011). We used the same presentation durations and interstimulus intervals as in our fMRI adaptation paradigm, and tested 10 participants, 6 of whom also participated in the fMRI experiment. We fitted the behavioral data with cumulative Gaussian functions to yield estimates of the point of subjective equality (PSE) which we used to illustrate the effect on numerosity adaptation on numerosity perception.

We found that, when compared to the control condition (no adaptation), there was a statistically significant increase in the perceived numerosity of the reference stimulus after adaptation to a low numerosity, and a statistically significant decrease in the perceived numerosity of the reference stimulus after adaptation to a high numerosity (Figure S2 in Supplementary Materials). Thus, our behavioral experiment confirms that the stimulus sequence we used in our fMRI experiment is able to elicit repulsive perceptual aftereffects in behaviorally susceptible numerosity ranges.

\section{4. fMRI acquisition}

We acquired MRI data on a 7T Philips Achieva scanner. Similar acquisition protocols are described fully in our previous studies (Harvey et al., 2015; Harvey and Dumoulin, 2017a). Briefly, we acquired T1-weighted anatomical scans, automatically segmented these with Freesurfer (http://freesurfer.net), then manually edited labels to minimize segmentation errors using ITK-SNAP (http://www.itksnap.org/). This provided a highly accurate cortical sur- face model at the gray-white matter border to characterize cortical organization. Functional T2*-weighted 2D echo planar images were acquired using multiband acquisition (multiband factor: 2 ) and anteriorposterior encoding, and a 32-channel head coil, at a resolution of $1.77 \times 1.77 \times 1.75 \mathrm{~mm}$, with a field of view of $227 \times 227 \times 70 \mathrm{~mm}$. The TR was $1400 \mathrm{~ms}$, echo time (TE) was $25 \mathrm{~ms}$, and flip angle was $70^{\circ}$ Functional runs were each 273 time frames (382.2 s) in duration, of which the first 9 time frames (12.6 s) were discarded to ensure the signal was at steady state.

Three scanning sessions were required for each participant. In each scanning session, 3 functional runs were acquired for the control condition ( 9 runs in total, total duration: $57.33 \mathrm{~min}$ ) and 3-4 runs for the adaptation conditions (10 runs for each adaptation condition in total, total duration: $63.70 \mathrm{~min}$; with the exception of one participant where 9 runs were acquired for each condition due to technical issues). The additional run we acquired for the adaptation conditions was done to ensure strong fMRI responses, because the main numerosity stimuli were replaced with the adapters in half of presentations. The order of the conditions was counterbalanced across runs within and between participants. Moreover, in each session we acquired a top-up scan recorded with the opposite phase-encoding direction to correct for image distortion in the gradient encoding direction (Andersson et al., 2003).

\subsection{Preprocessing of functional images}

Co-registration of functional data to the high-resolution anatomical space were performed using AFNI (afni.nimh.nih.gov; Cox, 1996), which differs from our previous studies. A single transformation matrix was constructed, incorporating all the steps from the raw data to the corti- 
cal surface model to reduce the number of interpolation steps to one. No other spatial or temporal smoothing procedures were applied. A T1 image with the same resolution, position and orientation as the functional data was first used to determine the transformation to a higher resolution (1 mm isotropic) whole-brain T1 image (3dUnifize, 3dAllineate). For the fMRI data, we first applied motion correction to two series of images that were acquired using opposing gradient encoding directions (3dvolreg). Subsequently, we determined the distortion transformation between the average images of these two series (3dQwarp). We then determined the transformation in head position between and within functional scans (3dNwarpApply). Then we determined the transformation that co-registers this functional data to the $\mathrm{T} 1$ acquired in the same space (3dvolreg). We applied the product of all these transformations at every TR to transform our functional data to the whole-brain T1 anatomy. We repeated this for each fMRI session to transform all their data to the same anatomical space. We then imported these data into Vistasoft's mrVista framework (github.com/vistalab/vistasoft) for analysis and model fitting. For each adaptation condition, the time series of separate scans were averaged together, resulting in a very high signal-to-noise ratio.

\section{6. $f M R I$ data analysis}

We estimated numerosity response models from fMRI data and stimulus time courses for each condition as previously described (Dumoulin and Wandell, 2008; Harvey et al., 2013, 2015; Harvey and Dumoulin, 2017a). The pRF model describes the aggregate tuning of the neural population within each fMRI recording site (voxel) using logarithmic Gaussian functions characterized by a preferred numerosity (mean of the Gaussian distribution) and a tuning width (standard deviation of the Gaussian in logarithmic numerosity space). A large set of candidate combinations of preferred numerosity and tuning width was generated.

At each gray matter voxel, the pRF model is estimated based on the fMRI data and the time course of numerosities presented within each TR. For each candidate preferred numerosity and tuning width, a predicted neural response time course is calculated as the amplitude of the candidate neural response function at each time point's presented numerosity. By convolving this predicted neural response time course with a hemodynamic response function (HRF), a predicted fMRI response time course is generated. For each fMRI recording site, the predicted fMRI response time course that most closely fits the recorded response time series course is chosen (by minimizing the sum of squared errors between the predicted and observed fMRI time series), giving the preferred numerosity and tuning width that generated that predicted fMRI response time course.

In the adaptation conditions, we fit models that include only the main numerosities, and models that include the main numerosities 1 through 7 and the presented adapter, and used the latter for subsequent analyses. However, in a general linear modeling framework (like a pRF model) these produce identical estimates of preferred numerosity and tuning width. Specifically, the adapter numerosity does not change through a scanning run, so it adds a constant component to the predicted response. FMRI data has an arbitrary baseline, so any constant component contributes to that baseline (which we do not analyze) without affecting other model parameters. We confirmed experimentally that $\mathrm{pRF}$ model parameters except baseline amplitude were identical whether we include or exclude the adapter state from the model's stimulus description. As a result of using this constant adapter, any changes in fMRI responses due to the presence of the adapter can only arise through non-linear interactions between response to the adapter and the main numerosity stimuli.

Candidate preferred numerosities extended beyond the presented numerosity range, allowing model fit parameters beyond this range. This meant that returned parameters within the 1 through 7 numerosity stimulus range were reported accurately, not just the best fit of a limited set. However, recording sites with preferences modeled outside the stimu- lus range must be treated with caution. In such recording sites, the response amplitude monotonically increases or decreases across the stimulus range. As such, we have little confidence that the preferred tuning estimate is correct. Therefore, recording sites whose preferred numerosity was outside the 1 through 7 range were excluded from further analysis.

Moreover, we excluded from further analysis the recording sites for which pRF models explained less than $27 \%$ of response variance (that is, those with a probability above $5 \%$ of observing this goodness of fit by chance). This threshold was calculated by fitting the numerosity models to the fMRI response time courses of white matter recording sites in the same scans for each participant. We then combined the numerosity models' fits from all participants' white matter to create a cumulative null distribution. The variance explained at the top $5 \%$ of that distribution (here, equivalent to $27 \%$ of response variance) was set as the threshold for all further gray matter analyses. Furthermore, and for all subsequent analyses, we selected recording sites for which pRF models explained more than $27 \%$ of response variance in the control condition alone. This meant that the selection of the recording sites were independent of their responses in the adaptation conditions.

\subsection{Definition of regions of interest (ROIs)}

We projected each recording site's preferred numerosity (estimated from the unadapted control condition) onto the inflated cortical surface for each condition. We defined region of interest (ROI) borders around numerosity maps showing a gradual change in numerosity preference, as previously described (Harvey and Dumoulin, 2017a).

In order to quantify changes in numerosity tuning between conditions, we examined the preferred numerosities of recording sites within each numerosity map and for each condition (control, low, and high numerosity adaptation). Specifically, we grouped the numerosity preferences of the recording sites in each numerosity map found in the left and right hemisphere across participants to create an aggregate pool of response data for each condition (control, low and high numerosity adaptation) in each numerosity map. We then selected the recording sites for which the pRF models explained more than $27 \%$ of response variance in the control condition. When comparing preferred numerosities between the control condition and either adaptation condition, we excluded recording sites with a preferred numerosity outside the presented numerosity range (1 through 7 ) in either compared condition.

In order to examine the effect of numerosity adaptation on neural numerosity preferences, we first calculated the change in preferred numerosity. For the low numerosity adaptation, we subtracted the preferred numerosity of recording sites in the low numerosity adaptation condition from the preferred numerosity of recording sites in the control condition. For the high numerosity adaptation, we subtracted the preferred numerosity of recording sites in the control condition from the preferred numerosity of recording sites in the high numerosity condition. Hence, in each adaptation condition, positive values indicate an attraction towards the numerosity of the adapter, and negative values indicate a repulsion from the numerosity of the adapter. We then binned the recording sites in each numerosity map into seven discrete numerosity categories ( 1 through 7 ) depending on their preferred numerosity in the control condition.

We also calculated the change in preferred numerosity by subtracting the preferred numerosity of recording sites in the control condition from the preferred numerosity of recording sites in each adaptation condition. Using this data representation, and in both adaptation conditions, positive versus negative values indicate a change in preferred numerosity towards higher versus lower preferred numerosities respectively, but each adapter induced a distinct effect (Figure S3 in Supplementary Materials). 


\section{a. Example fMRI time course}

\section{Control Condition}

Presented numerosity (n dots)

12345672020207654321202020

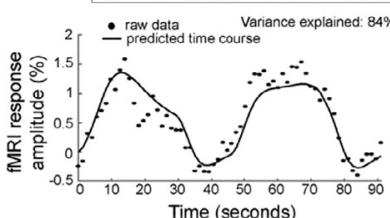

Time (seconds)

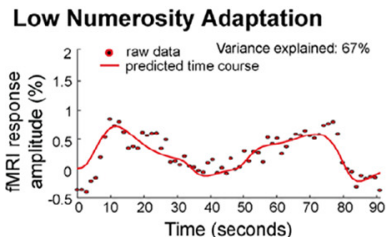

Time (seconds)

High Numerosity Adaptation

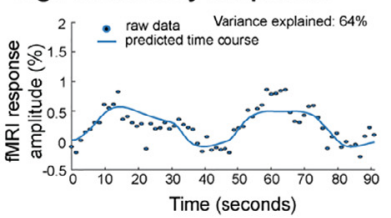

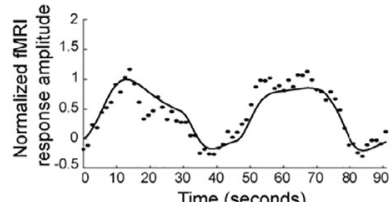

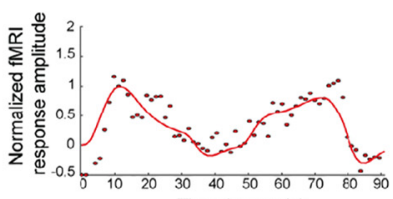

Time (seconds)

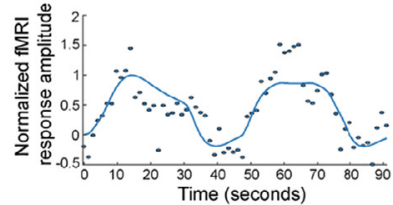

b. Neural response models

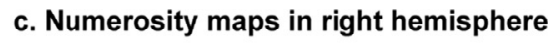

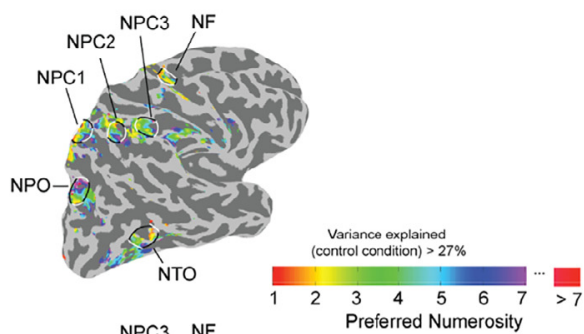
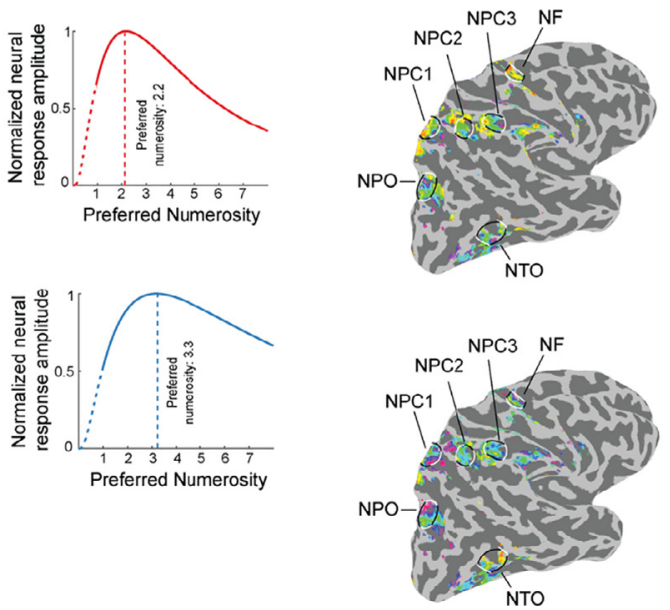

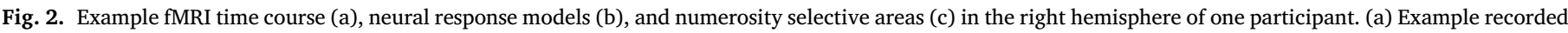

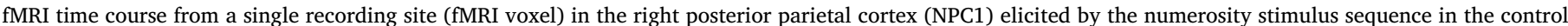

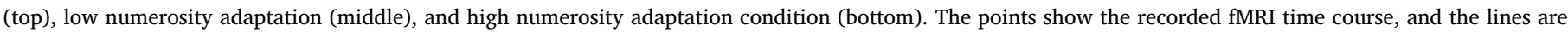

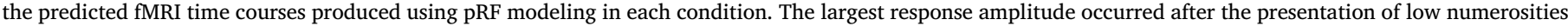

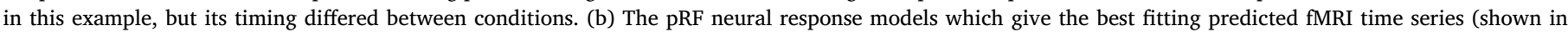

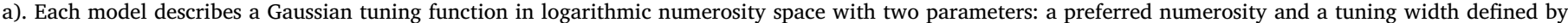

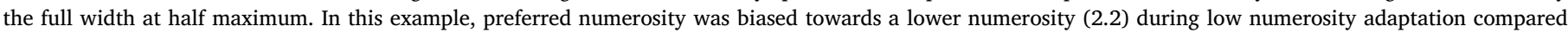

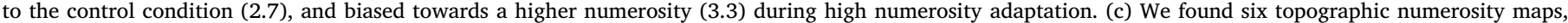

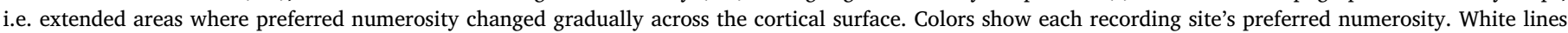

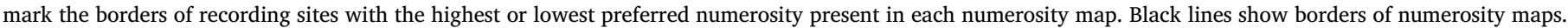

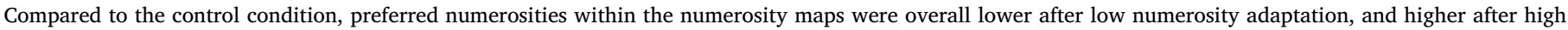
numerosity adaptation. (For interpretation of the references to color in this figure legend, the reader is referred to the web version of this article.)

\section{Results}

\subsection{FMRI response time courses and numerosity tuning functions change during adaptation}

As illustrated in Fig. 2, recorded fMRI response time courses at the same recording site differed in the three conditions (control, low numerosity adaptation, and high numerosity adaptation). The variance explained of the pRF models was lower in the adaptation conditions, as response amplitudes were lower. This is expected mainly due to less frequent presentation of the main (1 through 7 ) numerosity stimuli. The timing of responses also changed. The pRF models that best predict these responses capture these different responses as different preferred numerosity estimates in the three conditions.

\subsection{Replication of network of numerosity maps}

Numerosity maps were identified from the control condition (and clearly visible in the adaptation conditions) in the locations we have previously described (Harvey and Dumoulin, 2017a). NTO lay close to the temporo-occipital junction. NPO lay near the superior end of the parieto-occipital sulcus. NPC1, NPC2 and NPC3 lay in and around the parietal lobe's postcentral sulcus. NF lay at the junction of the precentral and superior frontal sulci. These locations were similar across participants (see Figure S4 and Table 1 in Supplementary Materials).

\subsection{Preferred numerosity is altered by numerosity adaptation}

As shown in Fig. 3a, during adaptation to a low numerosity (1 dot), the preferred numerosities of neural populations in NPC1 were typically lower than in the control condition, whereas during adaptation to a high numerosity ( 20 dots), the preferred numerosities of neural populations in NPC1 were typically higher than in the control condition (Fig. 3b).

\subsection{Preferred numerosity changes as a function of the unadapted preferred numerosity}

We found that after adaptation to a low numerosity, the higher range of preferred numerosities displayed attraction towards the numerosity of the adapter ( 1 dot), with the lower range of preferred numerosities displaying some repulsion from the numerosity of the adapter (Fig. 4). After adaptation to a high numerosity, we found that the higher range of preferred numerosities displayed repulsion from the numerosity of the adapter (20 dots), while the lower range displayed attraction towards the numerosity of the adapter (Fig. 4).

\subsection{Attraction of preferred numerosity towards the adapter numerosity increases with numerical distance from the adapter}

The differential attractive or repulsive changes in preferred numerosity we found in each adaptation condition could be attributed to the difference between the preferred numerosity in the control condition and 
NPC1

a.

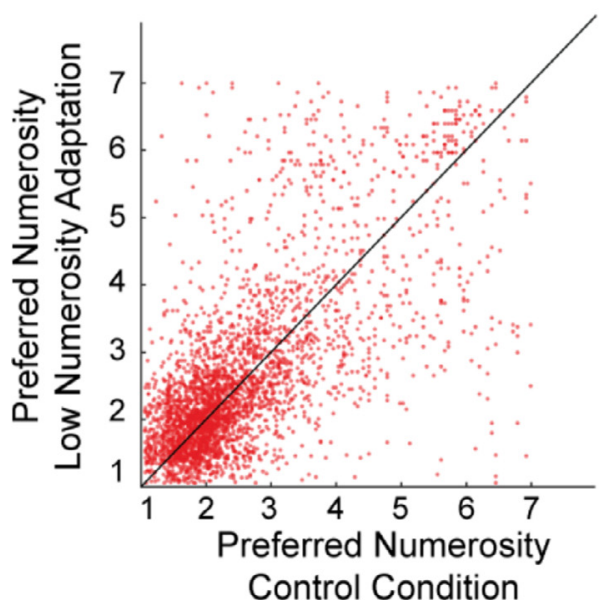

NPC1

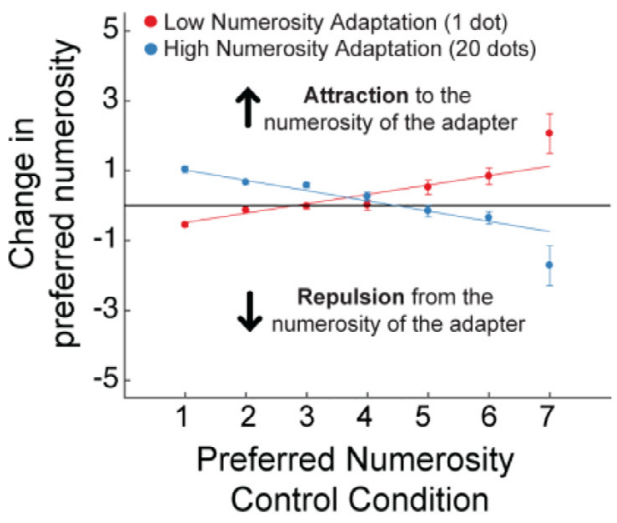

NPO

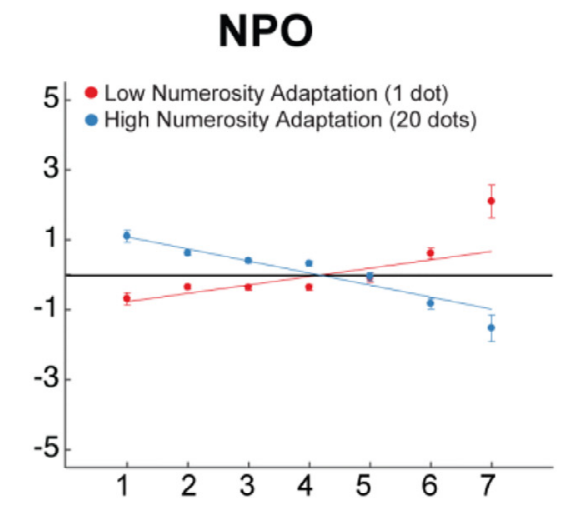

b.

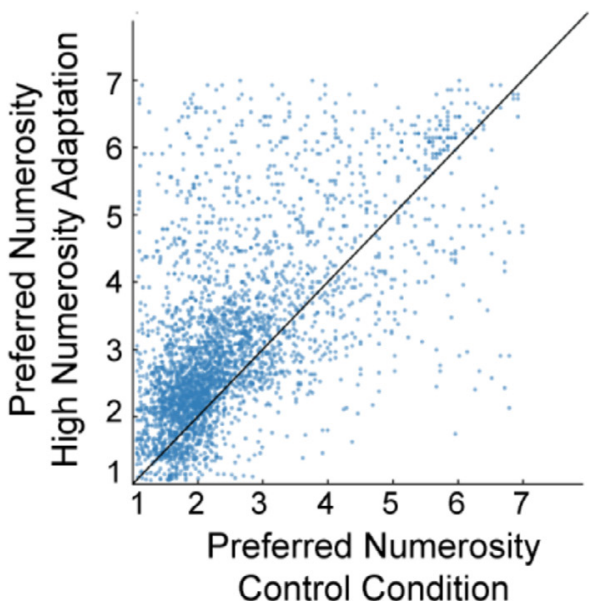

Fig. 3. Preferred numerosity of recording sites in NPC1 in the control vs. adaptation conditions. The recording sites illustrated correspond to the aggregate pool of recording sites in the leftand right-hemisphere NPC1 of all participants. (a) The preferred numerosity of recording sites was typically lower than in the control condition during low numerosity adaptation. (b) The preferred numerosity of recording sites was typically higher than in the control condition during high numerosity adaptation. (For interpretation of the references to color in this figure legend, the reader is referred to the web version of this article.)
NPC2
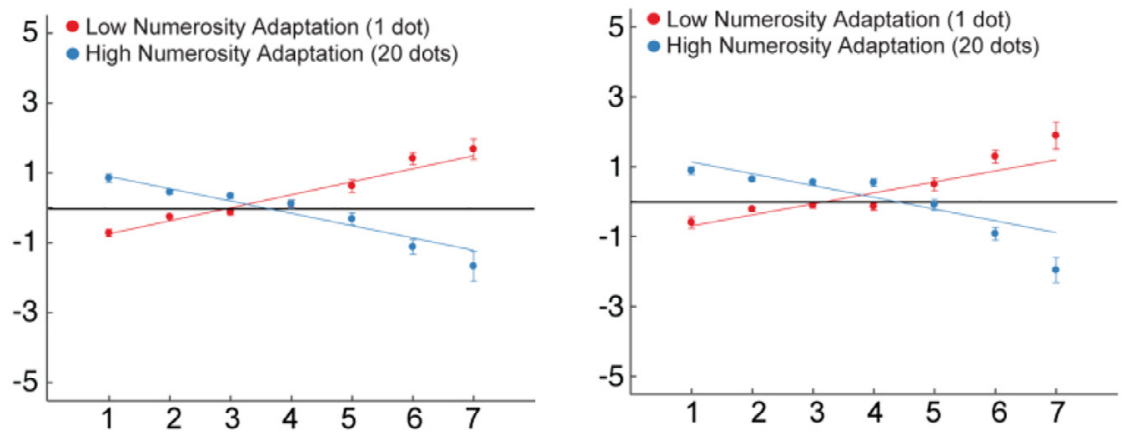
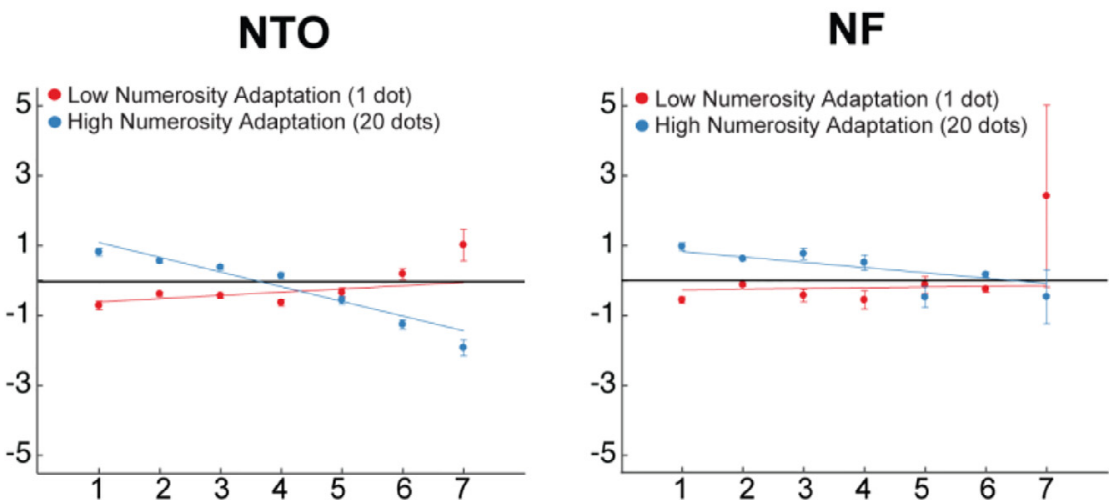

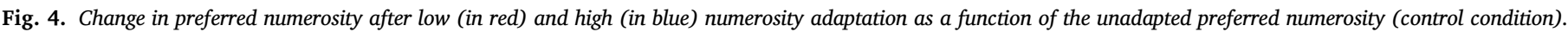

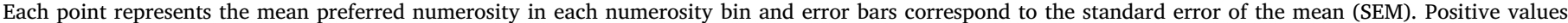

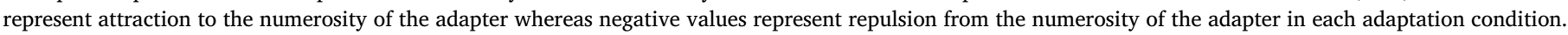

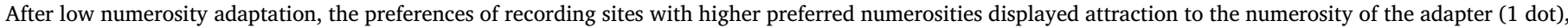

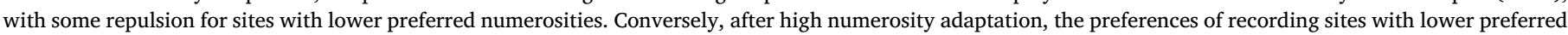

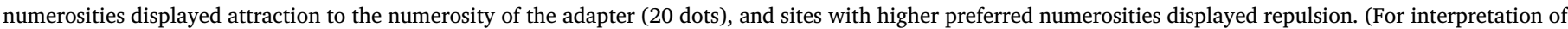
the references to color in this figure legend, the reader is referred to the web version of this article.) 


\section{NPC1}
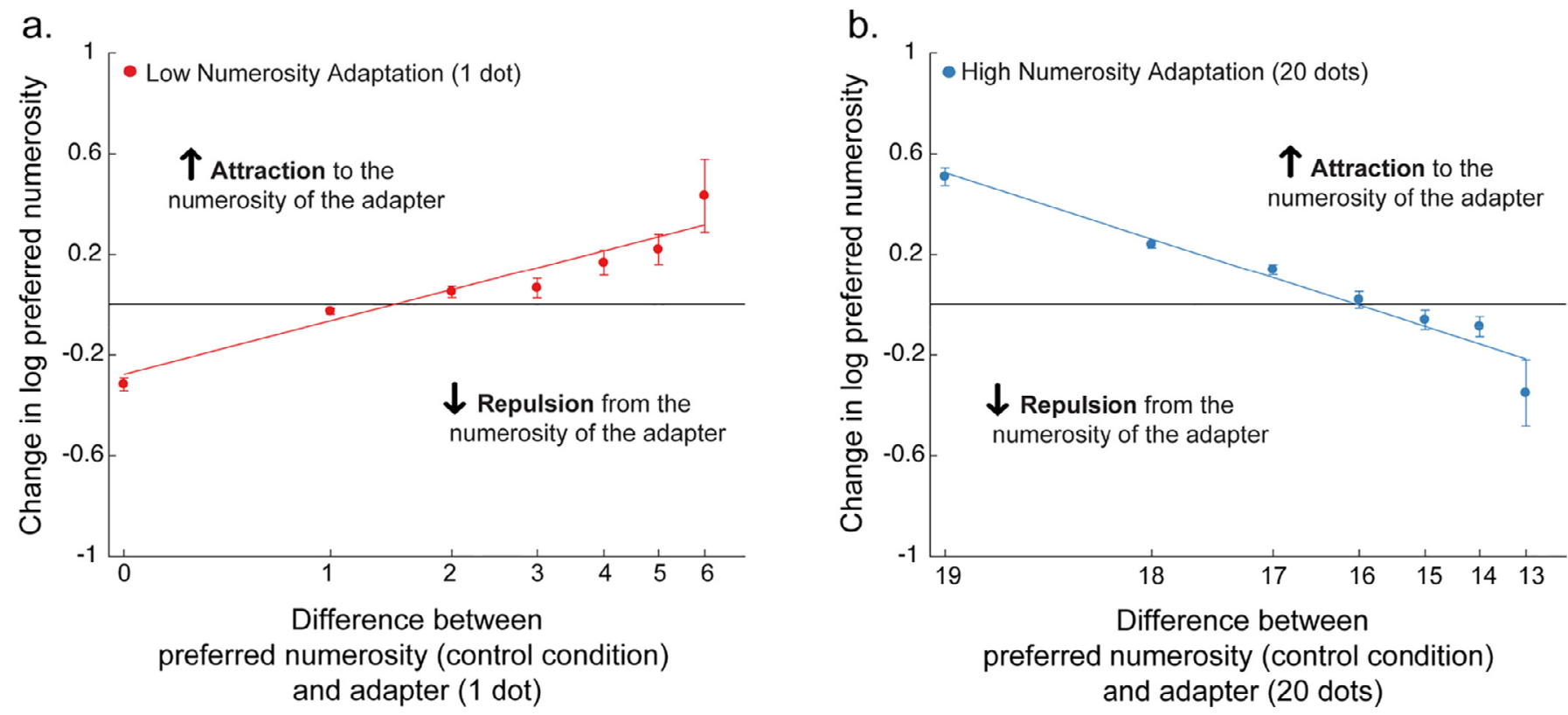

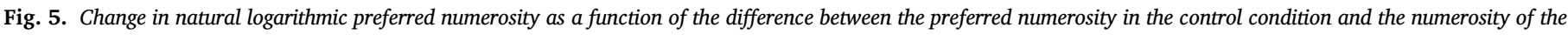

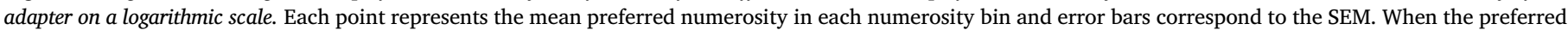

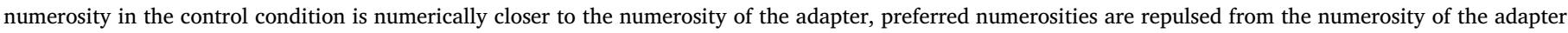

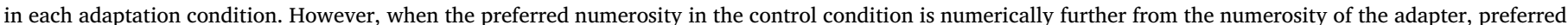

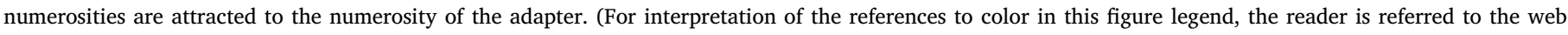
version of this article.)

the numerosity of the adapter. Fig. 5 illustrates the same data for NPC1 presented in Fig. 4, which are now in logarithmic numerosity space, with the $\mathrm{x}$ axis showing the difference between the preferred numerosity in the control condition and the numerosity of the adapter in each adaptation condition, and the $y$ axis showing the change in natural logarithmic preferred numerosity (see also Figure S5 in Supplementary Materials for each numerosity map). The choice of a logarithmic scale allowed for more homoscedastic data, resulting in more accurate linear fits that we subsequently performed (though the obtained results were similar to those obtained from when using linear numerosity space). This representation shows more explicitly that the attraction of preferred numerosity increases when preferred numerosities are numerically further from the numerosity of the adapter in each adaptation condition.

In order to further quantify the observed attraction to the numerosity of the adapter when preferred numerosities are numerically further from the numerosity of the adapter, we examined the change in natural logarithmic preferred numerosity as a function of the difference between the logarithmic preferred numerosity in the control condition and the logarithmic numerosity of the adapter. We did this for each adaptation condition, and for each numerosity map identified in each participant. Specifically, we first grouped the recording sites' data from the left- and right-hemisphere numerosity map of each participant. We then used a simple linear regression technique to fit the data of each numerosity map in each participant with a line and then used the slope of each regression line for subsequent analyses (Fig. 6 and S6 in Supplementary Materials for individual participants' data for each numerosity map).

We then did a group-level analysis to examine whether the slope values in each adaptation condition and numerosity map were significantly different from zero across participants (Fig. 7). We conducted a series of one-sample $t$-tests, after ensuring that the normality assumption was not violated by conducting a series of the Shapiro-Wilk tests $(p$ $>0.05$ for each variable), and used false discovery rate (FDR)-corrected significance thresholds.
When examining the mean slope values for the low numerosity adaptation condition, we found that they were significantly different from zero and this difference was statistically significant in all numerosity maps (NPC1: Mean $=0.36, S E=0.05, t(7)=7.29, p=0.001$; NPC2: Mean $=0.34, S E=0.04, t(7)=7.72, p=0.001$; NPC : Mean $=0.29, S E=$ $0.05, t(7)=5.58, p=0.001$; NPO: Mean $=0.35, S E=0.05, t(7)=7.51$, $p=0.001$; NTO: Mean $=0.23, S E=0.07, t(6)=3.41, p=0.014$; NF: Mean $=0.57, S E=0.12, t(7)=4.55, p=0.003$, FDR-corrected significance thresholds).

The same pattern was found in the high numerosity adaptation condition, where the mean slope values were significantly different from zero and this difference was statistically significant in all numerosity maps (NPC1: Mean $=-0.41, S E=0.08, t(7)=-5.25, p=0.002$; NPC2: Mean $=-0.45, S E=0.08, t(7)=-5.61, p=0.001$; NPC3: Mean $=-0.39$, $S E=0.04, t(7)=-9.10, p=0.001$; NP: Mean $=-0.44, S E=0.07, t(7)=$ $-6.64, p=0.001$; NTO: Mean $=-0.36, S E=0.06, t(6)=-5.63, p=0.002$; NF: Mean $=-0.62, S E=0.12, t(7)=-5.10, p=0.002$, FDR-corrected significance thresholds).

Furthermore, we obtained the same results when analyzing the slope values in each adaptation condition separately for the left- and righthemisphere numerosity maps (Figure S7 in Supplementary Materials).

We also tested whether the slope values in each adaptation condition were significantly different from zero across the numerosity maps identified in each participant (see Supplementary Materials). We conducted a series of one-sample $t$-tests and found that in all participants and adaptation conditions, the mean slope values were statistically different from zero, further validating the finding that preferred numerosity is attracted to the numerosity of the adapter when they are numerically further from each other (Figure S8 in Supplementary Materials).

\section{Discussion}

The goal of the present study was to examine whether and how the numerosity tuning of human neural populations changes during nu- 


\section{NPC1}

a.

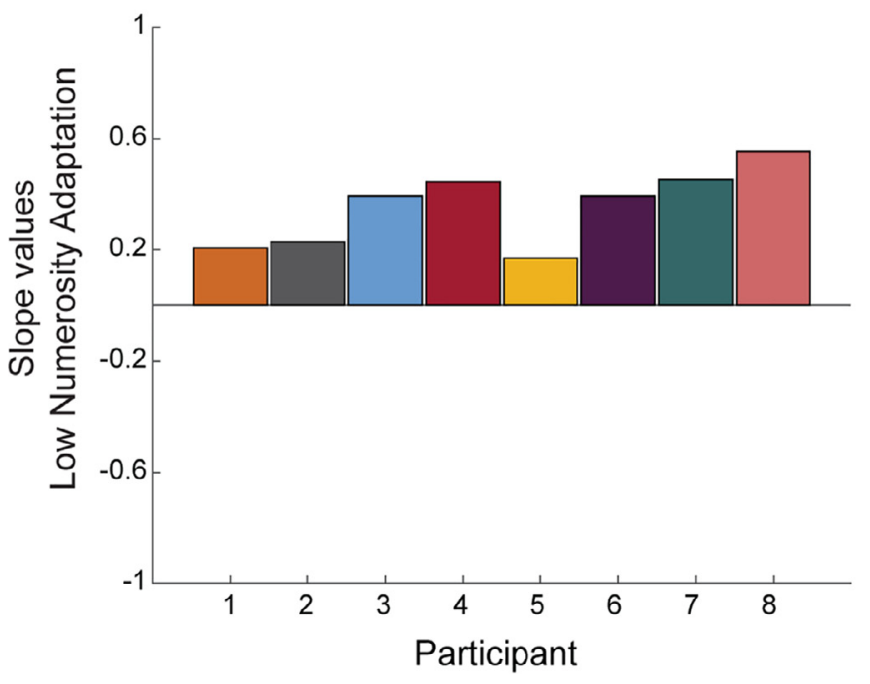

b.

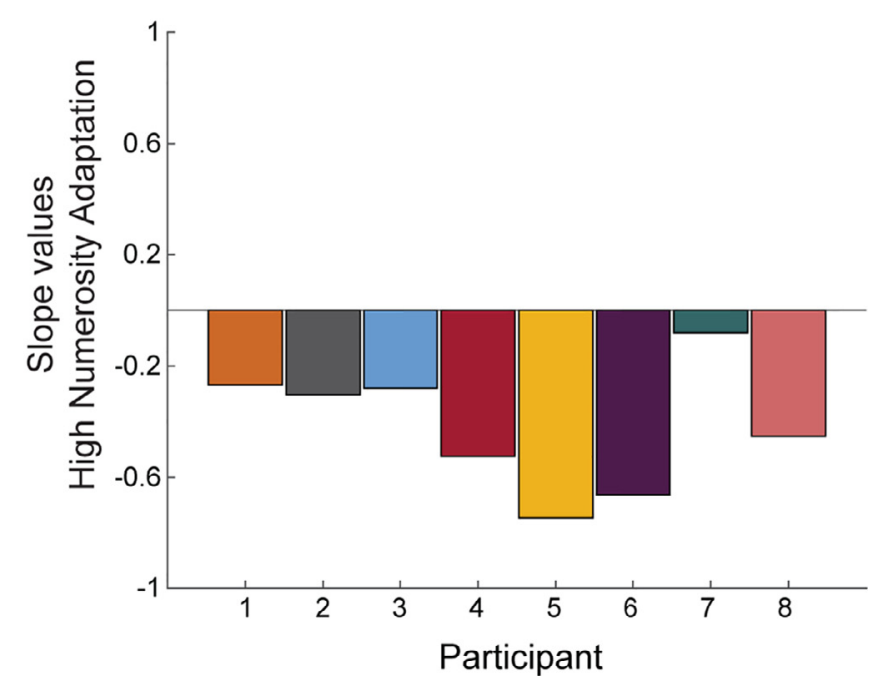

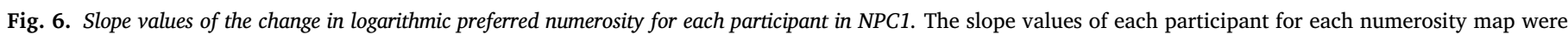

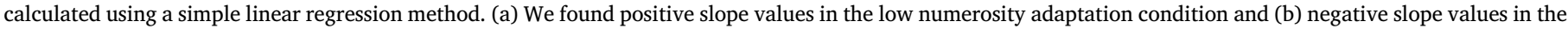

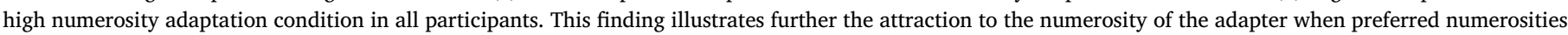

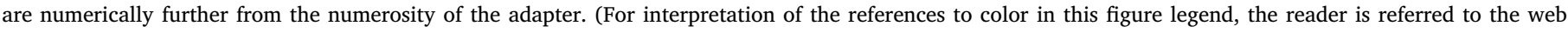
version of this article.)

merosity adaptation. We displayed visual stimuli of changing numerosity (1 through 7 ) which were interleaved with a low (1 dot) or high (20 dots) numerosity adapter while collecting ultra-high-field (7T) fMRI data. Using a pRF modeling approach, we quantified the preferred numerosity of neural populations during low and high numerosity adaptation and compared this to the preferred numerosity in an unadapted control condition. We focused on populations within six recently described topographic numerosity maps (Harvey and Dumoulin, 2017a). We found that numerosity preferences within each numerosity map decreased during low numerosity adaptation and increased during high numerosity adaptation, indicating that preferred numerosities were predominantly attracted to the numerosity of the adapter. However, when adapted to numerosity 1 , recording sites with preferred numerosities between 1 and 2 were (slightly) repelled from the adapted numerosity, and so were the recording sites with preferred numerosities between 6 and 7 when adapted to numerosity 20 .

In an effort to disentangle the attractive versus repulsive biases observed in each adaptation condition, we found that the observed biases could be explained in terms of the numerical distance of the unadapted preferred numerosities (control condition) from the numerosity of the adapter. Specifically, we found that preferred numerosities were attracted to the numerosity of the adapter when they were numerically further from the adapter in each adaptation condition. This effect of adaptation was markedly similar in all six numerosity maps found in our study. This is particularly striking given the generally wide structural separation of these areas across the cortical surface, and further reinforces the notion that these numerosity selective neural populations are part of a cohesive numerosity-processing network (Harvey and Dumoulin, 2017a). Specifically, it is possible that the different numerosity maps work in parallel to analyze different aspects of numerical information, similarly to how sensory maps operate (Young, 1998). Interestingly, the location of the maps includes areas implicated in a variety of perceptual and cognitive functions, among which are attentional control, decision-making, mathematics as well as visual motion, object processing and object recognition (Harvey and Dumoulin, 2017a). Furthermore, the NPC maps we find in the postcentral sulcus could be ho- mologues to the numerosity-selective neural populations identified on the fundus of the macaque intraparietal sulcus (Harvey et al., 2017; Nieder and Miller, 2004). Nevertheless, the causal role of these numerosity maps in numerical cognition remains to be established.

An fMRI study by Piazza et al. (2004), examining parietal responses to number change using a numerosity repetition suppression paradigm, found suppression of responses when the adapter and test stimuli were numerically close (e.g. both being 16 items), and recovery of responses when the test stimuli were $50 \%$ ( 8 items) or $200 \%$ (32 items) of the adapter (16 items). A similar effect was found by an fMRI repetition suppression study from Jacob and Nieder (2009), examining neural population tuning to preferred proportions (i.e. not absolute numerosity), where recovery from BOLD signal adaptation increased as the numerical distance between the adaptation and deviant proportions grew larger. In regard to our finding on the repulsion of preferred numerosity away from the adapter numerosity when they are numerically closer, it could be suggested that the response suppression reported by Piazza et al. (2004) and Jacob and Nieder (2009) could result in a repulsive change in preferred numerosity similar to the one we report here. Specifically, if part of the neural response function nearer the adapter numerosity is suppressed more than the part of the response function further away, the numerosity producing the largest response (the preferred numerosity) will move away from the adapter.

The overall attraction of neural numerosity tuning to the numerosity of the adapter is seemingly at odds with the repulsive aftereffects on numerosity perception during numerosity adaptation (Aagten-Murphy and Burr, 2016; Burr et al., 2011; Burr and Ross, 2008). However, we argue that the attractive biases on a neural level could potentially yield repulsive effects on perception. To give an illustrative example, adaptation to a numerosity of 1 might attract the unadapted preferred numerosity of a neural population from 5 to an adapted preferred numerosity of 4. Then, when 4 is presented, it will yield a maximal neural response in that population, while normally that maximal response would occur for 5 . In this way, the pattern of neural activity normally associated with 5 occurs for 4 , so 4 may be perceived as 5 , a repulsive effect on perception. 

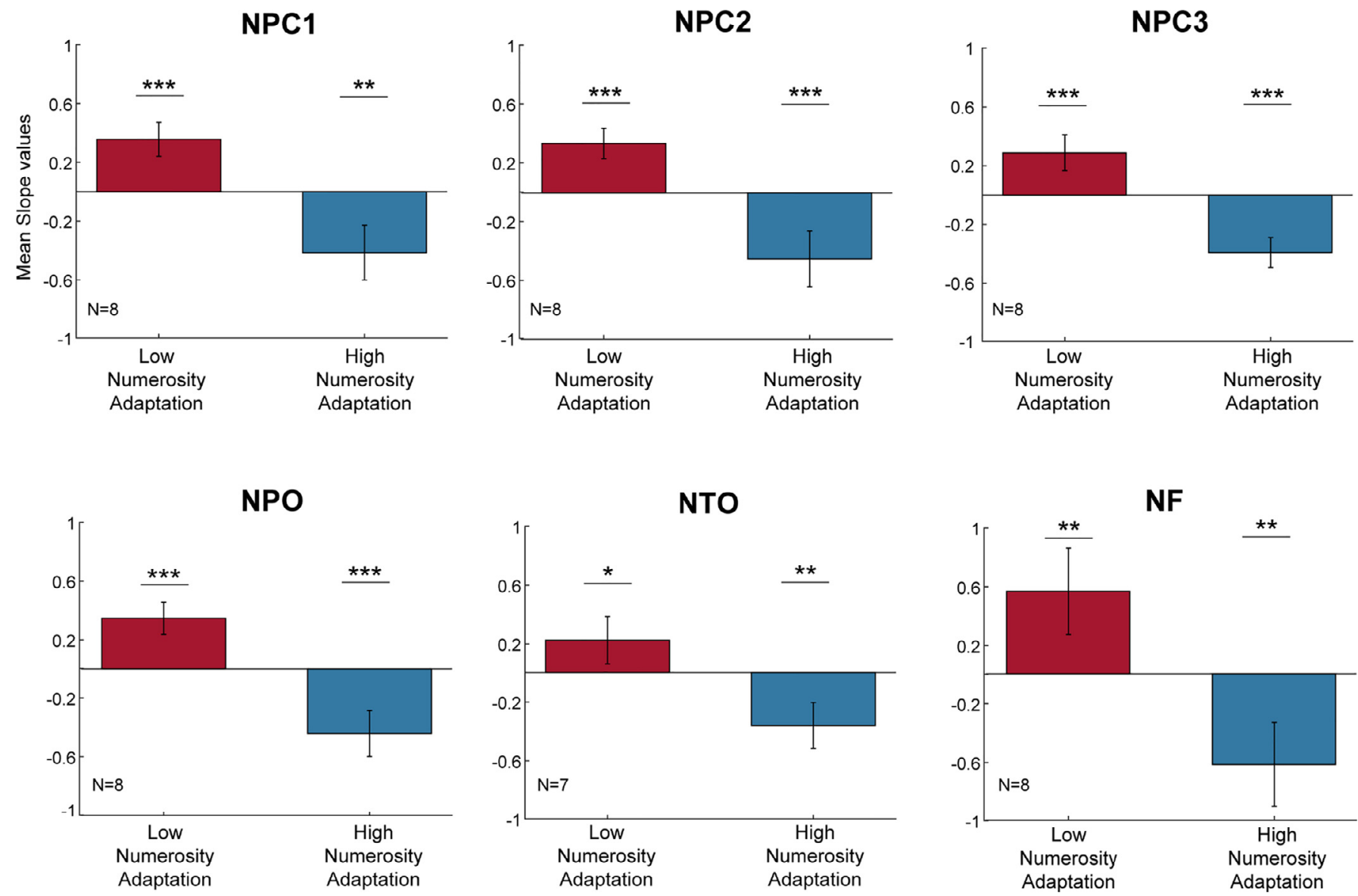

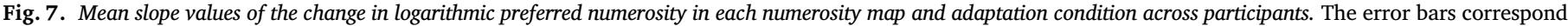

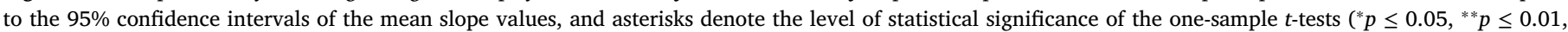

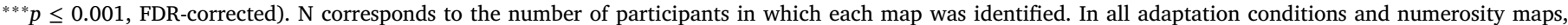

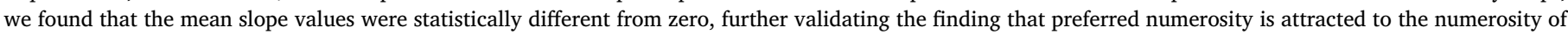

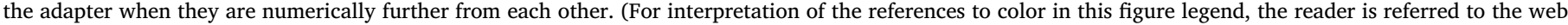
version of this article.)

Such links between attraction of neural response preferences and repulsion of perception have been described before. Kohn and Movshon (2004) examined how macaque MT motion direction tuning changes during motion direction adaptation. They modeled the response of the MT cell population in order to predict how the population response and thus, the perceived motion direction, would shift after adaptation. Their model showed that attractive shifts in single-cell tuning predict a repulsive perceptual aftereffect consistent with those measured psychophysically. We have previously shown that population receptive fields are attracted towards the focus of attention (Klein et al., 2014). Modeling these effects demonstrated that stimuli located near the focus of attention stimulate receptive fields that are positioned further away in the absence of attention (Klein et al., 2016). The perceived position of these stimuli shifts the stimulus away from the attended location, while population receptive fields shift towards the attended location.

Another possibility is that our stimulus could produce serial dependence rather than classical adaptation effects, yielding attraction rather than repulsion of perceived numerosity. Serial dependence attracts the perception of numerosity to the immediately preceding numerosity and operates over short timescales (Cicchini et al., 2014; Corbett et al., 2011; Fornaciai and Park, 2018). By conducting a validation experiment with our stimulus, we found evidence for repulsion (not attraction) of numerosity perception. This is in agreement with other psychophysical studies on numerosity adaptation using brief adapter presentation (Aagten-Murphy and Burr, 2016; Tsouli et al., 2019a,b). We are there- fore confident that our adaptation sequence repels numerosity while attracting neural numerosity preferences.

As we note in the Methods, our stimulus sequence presented the same numerosity repeatedly and frequently (every $700 \mathrm{~ms}$ ) and moved through numerosities gradually and sequentially (every $4200 \mathrm{~ms}$ ), as in our previous experiments. This repeated presentation and gradual change are likely to cause some repetition suppression of responses to each stimulus display, and perhaps perceptual adaptation to the changing numerosity as well as the adaptors. First, while repetition suppression will reduce the responses to each display, presenting each numerosity only once before moving on would almost certainly produce a smaller response and less clear results. Second, given the slowness of the hemodynamic response and resulting poor temporal resolution of fMRI, changing numerosity on every display would prevent us from separating responses to different numerosities in the resulting fMRI time course (if presented at the same rate) or would require a few seconds between displays, and therefore far more scanning. These design choices greatly increase the efficiency of our method to quantify numerosity selectivity using fMRI.

But how do these factors affect our estimates of preferred numerosity? First, changing numerosities slowly and sequentially makes the preferred numerosity estimates less sensitive to the precise timing of the modeled hemodynamic response function: small deviations from the modeled timing only slightly change which numerosity was being presented at the time of any modeled neural response. Second, we do not 
expect repeated presentations and slow, sequential changes to systematically affect preferred numerosity estimates. Repetition suppression of responses from the same numerosity should not change which part of the response function is responding, only reduce its amplitude. A preceding presentation of a different numerosity is likely to affect the preferred numerosity estimate, but by using "sweeps" in both ascending and descending directions for the same model we aim to balance opposing effects of preceding lower and higher numerosities. Given that we find that the adaptation's neural effects increase with distance between the adapter's numerosity and the population's preferred numerosity, keeping preceding numerosities as close as possible to the current presentation should also minimize effects of preferred numerosity estimates. Finally, it is likely that participants know which numerosity to expect in each display, as this is highly predictable. It is less clear how expectation might affect numerosity selectivity, but we would expect a global increase or decrease in response amplitude and we have no reason to expect a systematic bias would result from expectation. Importantly, all of the effects described above should affect the responses to both adaptation conditions similarly, so seem unlikely to explain the differences we see between conditions.

Our results further illustrate that the preferred numerosity of neural populations with numerosity preferences in the subitizing range (less than 4, allowing fast and error-free numerosity judgements) can be affected by adaptation. The majority of psychophysical studies on numerosity adaptation (Aagten-Murphy and Burr, 2016; Anobile et al., 2018; Burr and Ross, 2008; Liu et al., 2017; Tsouli et al., 2019a,b) report effects on perception of higher numerosities (typically $\geq 9$ items). Only one behavioral study has shown adaptation effects on perception of numerosities within the subitizing range, and only under conditions of high attentional load induced by a secondary task (Burr et al., 2011). Here, we show that neural numerosity tuning within the subitizing range can be altered by numerosity adaptation even in the absence of attentional load. Nevertheless, it is possible that this change in neural tuning for numerosities within the subitizing range might be too subtle to affect numerosity perception when measured psychophysically.

\section{Conclusions}

Using a numerosity adaptation paradigm combined with ultra-high field fMRI and a pRF modeling approach, we show that neural numerosity selectivity was altered systematically in all numerosity maps. Specifically, neural numerosity preferences were overall attracted to the numerosity of the adapter, with the extent of attraction increasing when the unadapted preferred numerosities were numerically further from the numerosity of the adapter. We argue that these attractive biases could potentially underlie the perceptual effects of numerosity adaptation.

\section{Acknowledgments}

This work was supported in part by the AMMODO KNAW Award (SD). The Spinoza Centre for Neuroimaging is a joint institute of the University of Amsterdam, Academic Medical Center, VU University, VU Medical Center, Netherlands Institute for Neuroscience and the Royal Netherlands Academy of Sciences.

\section{Data and code availability statement}

The code generated during this study is available in the Vistasoft repository (https://github.com/vistalab/vistasoft).

The datasets supporting the current study are available from the corresponding author on request. The datasets have not yet been deposited in a public repository because of (biometric) data protection issues, in compliance with the General Data Protection Regulation (GDPR; https://ec.europa.eu/info/law/law-topic/dataprotection/data-protection-eu_en) for data protection in the European Union, and in compliance with the data sharing policy as reviewed by the Medical Ethical Review Committee of the University Medical Center Utrecht.

\section{Author contributions}

Conceptualization: A.T., B.M.H., S.F.T.P., M.J.V.D.S., and S.O.D.; Methodology: A.T., B.M.H., S.F.T.P., M.J.V.D.S., and S.O.D.; Software: M.V.A., B.M.H., and S.O.D.; Validation: A.T., Y.C., M.V.A., S.H., B.M.H., and S.O.D.; Formal Analysis: A.T., Y.C., S.H., B.M.H., S.F.T.P., M.J.V.D.S., and S.O.D.; Investigation: A.T. and Y.C.; Data Curation: A.T., Y.C., and M.V.A.; Writing - Original Draft: A.T.; Writing - Review \& Editing: A.T., Y.C., M.V.A., S.H., B.M.H., S.F.T.P., M.J.V.D.S., and S.O.D.; Visualization: A.T., Y.C., S.H., and B.M.H.; Supervision: B.M.H., S.F.T.P., M.J.V.D.S., and S.O.D.; Project administration: B.M.H., S.F.T.P., M.J.V.D.S., and S.O.D.; Funding Acquisition: S.O.D.

\section{Supplementary materials}

Supplementary material associated with this article can be found, in the online version, at doi:10.1016/j.neuroimage.2021.117794.

\section{References}

Aagten-Murphy, D., Burr, D., 2016. Adaptation to numerosity requires only brief exposures, and is determined by number of events, not exposure duration. J. Vis. 16 (10), 22. doi:10.1167/16.10.22.

Andersson, J.L.R., Skare, S., Ashburner, J, 2003. How to correct susceptibility distortions in spin-echo echo-planar images: application to diffusion tensor imaging. Neuroimage 20 (2), 870-888. doi:10.1016/S1053-8119(03)00336-7.

Anobile, G., Cicchini, G.M., Burr, D., 2016. Number as a primary perceptual attribute: a review. Perception 45 (1-2), 5-31. doi:10.1177/0301006615602599.

Anobile, G., Cicchini, G.M., Gasperini, F., Burr, D.C., 2018. Typical numerosity adaptation despite selectively impaired number acuity in dyscalculia. Neuropsychologia $120,43-$ 49. doi:10.1016/j.neuropsychologia.2018.10.006.

Brainard, D.H., 1997. The psychophysics toolbox. Spat. Vis. 10 (4), 433-436. doi:10.1163/156856897X00357.

Burr, D., Anobile, G., Arrighi, R., 2017. Psychophysical evidence for the number sense. Philos. Trans. R. Soc. B: Biol. Sci. 373 (1740). doi:10.1098/rstb.2017.0045, 20170045.

Burr, D., Anobile, G., Turi, M., 2011. Adaptation affects both high and low (subitized) numbers under conditions of high attentional load. Seeing Perceiv. 24 (2), 141-150. doi:10.1163/187847511X570097.

Burr, D., Ross, J., 2008. A visual sense of number. Curr. Biol. 18 (6), 425-428. doi:10.1016/j.cub.2008.02.052.

Cantlon, J.F., Brannon, E.M., Carter, E.J., Pelphrey, K.A., 2006. Functional imaging of numerical processing in adults and 4-y-old children. PLoS Biol. 4 (5), e125. doi:10.1371/journal.pbio.0040125.

Castaldi, E., Aagten-Murphy, D., Tosetti, M., Burr, D., Morrone, M.C., 2016. Effects of adaptation on numerosity decoding in the human brain. Neuroimage 143, 364-377. doi:10.1016/j.neuroimage.2016.09.020.

Cicchini, G.M., Anobile, G., Burr, D.C., 2014. Compressive mapping of number to space reflects dynamic encoding mechanisms, not static logarithmic transform. Proc. Natl. Acad. Sci. 111 (21), 7867-7872. doi:10.1073/pnas.1402785111.

Corbett, J.E., Fischer, J., Whitney, D., 2011. Facilitating stable representations: serial dependence in vision. PLoS ONE 6 (1), e16701. doi:10.1371/journal.pone.0016701.

Cox, R.W., 1996. AFNI: software for analysis and visualization of functional magnetic resonance neuroimages. Comput. Biomed. Res. 29 (3), 162-173. doi:10.1006/cbmr.1996.0014.

Demeyere, N., Rotshtein, P., Humphreys, G.W., 2014. Common and dissociated mechanisms for estimating large and small dot arrays: value-specific fMRI adaptation. Hum. Brain Mapp. 35 (8), 3988-4001. doi:10.1002/hbm.22453.

Dumoulin, S.O., Wandell, B.A., 2008. Population receptive field estimates in human visual cortex. Neuroimage 39 (2), 647-660. doi:10.1016/j.neuroimage.2007.09.034.

Fornaciai, M., Park, J., 2018. Serial dependence in numerosity perception. J. Vis. 18 (9), 15. doi:10.1167/18.9.15, -15 .

Grill-Spector, K., Henson, R., Martin, A., 2006. Repetition and the brain: neural models of stimulus-specific effects. Trends Cogn. Sci. 10 (1), 14-23. doi:10.1016/j.tics.2005.11.006.

Harvey, B.M., Dumoulin, S.O., 2017a. A network of topographic numerosity maps in human association cortex. Nat. Hum. Behav. 1, 36. doi:10.1038/s41562-016-0036.

Harvey, B.M., Dumoulin, S.O., 2017b. Can responses to basic non-numerical visual features explain neural numerosity responses? Neuroimage 149, 200-209. doi:10.1016/j.neuroimage.2017.02.012.

Harvey, B.M., Ferri, S., Orban, G.A., 2017. Comparing parietal quantity-processing mechanisms between humans and macaques. Trends Cogn. Sci. 21 (10), 779-793. doi:10.1016/j.tics.2017.07.002.

Harvey, B.M., Fracasso, A., Petridou, N., Dumoulin, S.O., 2015. Topographic representations of object size and relationships with numerosity reveal generalized quantity processing in human parietal cortex. Proc. Natl. Acad. Sci. 112 (44), 13525-13530. doi:10.1073/pnas.1515414112. 
Harvey, B.M., Klein, B.P., Petridou, N., Dumoulin, S.O., 2013. Topographic Representation of Numerosity in the Human Parietal Cortex. Science 341 (6150), 1123-1126. doi:10.1126/science.1239052.

He, L., Zhou, K., Zhou, T., He, S., Chen, L., 2015. Topology-defined units in numerosity perception. Proc. Natl. Acad. Sci. 112 (41), E5647-E5655. doi:10.1073/pnas.1512408112.

Howard, S.R., Avarguès-Weber, A., Garcia, J.E., Greentree, A.D., Dyer, A.G., 2018. Numerical ordering of zero in honey bees. Science 360 (6393), 1124-1126. doi:10.1126/science.aar4975.

Izard, V., Sann, C., Spelke, E.S., Streri, A., 2009. Newborn infants perceive abstract numbers. Proc. Natl. Acad. Sci. 106 (25), 10382-10385. doi:10.1073/pnas.0812142106.

Jacob, S.N., Nieder, A., 2009. Tuning to non-symbolic proportions in the human frontoparietal cortex: representation of proportions in the human brain. Eur. J. Neurosci. 30 (7), 1432-1442. doi:10.1111/j.1460-9568.2009.06932.x.

Kersey, A.J., Cantlon, J.F., 2017. Neural tuning to numerosity relates to perceptual tuning in 3-6-year-old children. J. Neurosci. 37 (3), 512-522. doi:10.1523/JNEUROSCI.0065-16.2016.

Klein, B.P., Harvey, B.M., Dumoulin, S.O., 2014. Attraction of Position Preference by Spatial Attention throughout Human Visual Cortex. Neuron 84 (1), 227-237. doi:10.1016/j.neuron.2014.08.047.

Klein, B.P., Paffen, C.L.E., Pas, S.F.te, Dumoulin, S.O, 2016. Predicting bias in perceived position using attention field models. J Vis 16 (7), 15. doi:10.1167/16.7.15.

Kleiner, M., Brainard, D.H., Pelli, D., Ingling, A., Murray, R., Broussard, C., 2007. What's new in psychtoolbox-3. Perception 36, 1-16. doi:10.1068/v070821.

Kohn, A., Movshon, J.A., 2004. Adaptation changes the direction tuning of macaque MT neurons. Nat. Neurosci. 7 (7), 764-772. doi:10.1038/nn1267.

Krekelberg, B., Boynton, G.M., van Wezel, R.J.A., 2006. Adaptation: from single cells to BOLD signals. Trends Neurosci. 29 (5), 250-256. doi:10.1016/j.tins.2006.02.008.

Krusche, P., Uller, C., Dicke, U., 2010. Quantity discrimination in salamanders. J. Exp. Biol. 213 (11), 1822-1828. doi:10.1242/jeb.039297.

Kutter, E.F., Bostroem, J., Elger, C.E., Mormann, F., Nieder, A., 2018. Single neurons in the human brain encode numbers. Neuron 100 (3), 753-761. doi:10.1016/j.neuron.2018.08.036, e4.

Larsson, J., Solomon, S.G., Kohn, A., 2016. FMRI adaptation revisited. Cortex 80, 154-160. doi:10.1016/j.cortex.2015.10.026.

Liu, W., Zhang, Z.-.J., Zhao, Y.-.J., Li, B.-.C., Wang, M., 2017. Distinct mechanisms in the numerosity processing of random and regular dots. Acta Psychol. (Amst) 174, 17-30. doi:10.1016/j.actpsy.2017.01.006.
Miletto Petrazzini, M.E., Agrillo, C., Izard, V., Bisazza, A., 2016. Do humans (Homo sapiens) and fish (Pterophyllum scalare) make similar numerosity judgments? J. Comp. Psychol. 130 (4), 380-390. doi:10.1037/com0000045.

Nieder, A., Freedman, D.J., Miller, E.K., 2002. Representation of the quantity of visual items in the primate prefrontal cortex. Science 297 (5587), 1708-1711. doi:10.1126/science.1072493.

Nieder, A., Miller, E.K., 2003. Coding of cognitive magnitude: compressed scaling of numerical information in the primate prefrontal cortex. Neuron 37 (1), 149-157. doi:10.1016/S0896-6273(02)01144-3.

Nieder, A., Miller, E.K., 2004. A parieto-frontal network for visual numerical information in the monkey. Proc. Natl. Acad. Sci. 101 (19), 7457-7462. doi:10.1073/pnas.0402239101.

Piazza, M., Izard, V., Pinel, P., Le Bihan, D., Dehaene, S., 2004. Tuning curves for approximate numerosity in the human intraparietal sulcus. Neuron 44 (3), 547-555. doi:10.1016/j.neuron.2004.10.014.

Roggeman, C., Santens, S., Fias, W., Verguts, T., 2011. Stages of nonsymbolic number processing in occipitoparietal cortex disentangled by fMRI adaptation. J. Neurosci. 31 (19), 7168-7173. doi:10.1523/JNEUROSCI.4503-10.2011.

Scarf, D., Hayne, H., Colombo, M., 2011. Pigeons on Par with Primates in Numerical Competence. Science 334 (6063), 1664. doi:10.1126/science.1213357, -1664.

Shuman, M., Kanwisher, N., 2004. Numerical magnitude in the human parietal lobe: tests of representational generality and domain specificity. Neuron 44 (3), 557-569. doi:10.1016/j.neuron.2004.10.008.

Tsouli, A., Dumoulin, S.O., te Pas, S.F., van der Smagt, M.J., 2019a. Adaptation reveals unbalanced interaction between numerosity and time. Cortex 114, 5-16. doi:10.1016/j.cortex.2018.02.013.

Tsouli, A., van der Smagt, M.J., Dumoulin, S.O., Te Pas, S.F., 2019b. Distinct temporal mechanisms modulate numerosity perception. J. Vis. 19 (6), 19. doi:10.1167/19.6.19.

Viswanathan, P., Nieder, A., 2013. Neuronal correlates of a visual "sense of number" in primate parietal and prefrontal cortices. Proc. Natl. Acad. Sci. 110 (27), 11187-11192. doi:10.1073/pnas.1308141110.

Wagener, L., Loconsole, M., Ditz, H.M., Nieder, A., 2018. Neurons in the endbrain of numerically naive crows spontaneously encode visual numerosity. Curr. Biol. 28 (7), 1090-1094. doi:10.1016/j.cub.2018.02.023.

Young, E.D., 1998. Parallel processing in the nervous system: evidence from sensory maps. PNAS 95 (3), 933-934. doi:10.1073/pnas.95.3.933, PubMed.. 